\title{
TITLE: Dignity, dining and dialogue: reviewing the literature on quality of life for people with dementia
}

\author{
AUTHOR: \\ Dr Lorraine Venturato \\ Research Centre for Clinical and Community Practice Innovation, \\ Griffith Health Institute, \\ Griffith University, \\ Kessels Road, \\ Nathan QLD 4111, \\ AUSTRALIA \\ Email: I.venturato@griffith.edu.au
}

\begin{abstract}
:
Aim. To review contemporary literature on quality of life for people with dementia.

Background. One of the consequences of population ageing is the estimated increase in the incidence of dementia. While we can as yet do little to affect the course of the disease, health care professionals are well-placed to intervene to affect quality of life and quality of care for people with dementia.
\end{abstract}

Results. Research into quality of life for people with dementia has grown markedly over the last decade, in line with evolving conceptual and theoretical understandings of this complex concept. While much of the literature continues to focus on assessment and measurement of quality of life, emerging areas of interest are noted, including dialogue associated with personhood; dignity; and dining and mealtime experiences.

Conclusion. Literature on quality of life for people with dementia is as diverse as the concept itself. Established and emerging areas of research suggest that this diversity will continue into the future as we strive to better understand quality of life for people with dementia in all its complexity.

Key Words: quality of life; dementia; literature review; personhood; dignity; dining; mealtimes 


\section{INTRODUCTION}

Along with the ageing demographic profile of both developing and developed nations, comes an increase in the prevalence rates of age associated conditions, such as dementia. Current global estimates suggest there are 24.3 million people living with dementia, and that there will be 4.6 million new cases diagnosed worldwide each year (Ferri et al. 2005). It is estimated that, on current mortality and burden rates, up to 71 percent of these cases will be in the developing world (Kalaria et al. 2008). Such increases are significant as people with dementia (PWD) tend to be heavy consumers of health care services, and dementia care is both time and labour intensive as a result of the degenerative impairments and cognitive changes associated with dementia (Ferri et al. 2005). Global Burden of Disease Estimates suggest that the disability weight or burden for dementia is high (World Health Organisation 2003), with community and residential care bearing the brunt of direct costs (Ferri et al. 2005). There are also significant indirect costs associated with dementia however, with informal or family caregivers an important part of the care and support system for people living with dementia.

The growing prevalence, together with the significant economic, personal, and social costs associated with dementia, has ensured dementia is a recognised health and research priority in many developed nations. Governments, health care providers and inter/national Alzheimer's Associations promote a two pronged clinical agenda, aimed at both medically-driven interventions and treatments, and social and psychological support for PWD and their caregivers. Indeed, while medical research continues to strive towards treatment and cure, other health and social science research is focused on enhancing the quality of life and quality of care for those living with dementia today. This review of the literature is particularly focused on the later, that is, the body of clinical, conceptual and research-based work that focuses on enhancing quality of life for people living with dementia. 


\section{Quality of Life (QOL)}

QOL is a complex, multidimensional construct, which includes physical, psychological, and social components (Birren, Lubben \& Rowe, 1991) and, in people with dementia, significant cognitive components (Murray \& Boyd, 2009). It encompasses both objective (eg. level of income, education, housing) and subjective (eg. well-being, dignity) domains; macro (social) and micro (individual) aspects; and positive and negative components. Together, these interact to produce what is essentially an open-ended and individualistic concept. As Bond (1999, p. 566) notes, QOL has no clear or fixed boundary and "there is little agreement about what constitutes the individual 'domains' of quality of life; about the standard for each 'domain' which would reflect a low or high quality of life; or who determines the relevance of each 'domain' to the individual". He suggests a broad range of relevant domains including health status characteristics, clinical characteristics, physical environmental factors, social environmental factors, socio-economic factors, personal autonomy factors, subjective satisfaction, personality factors and cultural factors. Other domains identified within the literature include dignity, autonomy, choice, control, privacy, as well as a sense of safety, security and order, physical comfort, enjoyment, meaningful activity, relationships, spiritual well-being, functional competence, self-determination, participation, and accommodation of needs (Courtney et al. 2003; Kane et al. 2003).

Given the breadth and variety of domains and concepts associated with QOL it is not surprising perhaps that there is no single agreed or universally accepted definition of QOL (Walker \& Lowenstein, 2009). However, a widely used generic definition has been developed by the World Health Organisation reflecting many of the domains suggested above:

Quality of life is defined as an individual's perceptions of their position in life in the context of the culture and values systems in which they live and in relation to their goals, expectations, standards and concerns. It is a broad ranging concept, affected in a complex way by a person's physical 
health, psychological state, personal beliefs, social relationships and their relationship to their environment.

(WHO, 2004)

Dementia and cognitive impairment may have a profound effect on many of the domains inherent in the above definition and definitions of $\mathrm{QOL}$ for people with dementia often reflect the conceptualisation and framework of the researcher.

Murray and Boyd (2009) note that QOL for PWD is further complicated by the blending of medical and social care and constructions of QOL, as well as the inherent complexity of QOL and the heterogeneity of people with dementia. This review recognises all of these features and acknowledges that $\mathrm{QOL}$ is essentially a unique, individually defined construct (important for understanding and clinical care of individuals), while at the same time acknowledging the need to consider and evaluate QOL at a group level (important for intervention studies and health service research).

\section{AIMS}

This paper aims to review the literature on QOL for people living with dementia across care settings in order to shed light on contemporary understandings and research developments in this area.

\section{METHODS}

A review of contemporary literature on $\mathrm{QOL}$ for PWD was undertaken for this paper. The review focused on peer-reviewed journals, with articles published in English between 1999 and 2009. Literature sourced included conceptual, clinical and research articles. A broad approach was undertaken in this review in order to explore current research and clinical issues, and emerging trends in thinking about QOL and PWD. Inclusion and exclusion criteria included:

\section{Inclusion Criteria:}

- focus on quality of life issues for people living with dementia;

- published in peer-reviewed journals between 1999 and 2009. 


\section{Exclusion Criteria:}

- quality of life papers not focused on people living with dementia;

- grey literature or non peer-reviewed publications.

\section{Search Strategy:}

A search was conducted using the terms 'quality of life'; 'dementia'; and 'older people'; across three databases: Cumulative Index of Nursing and Allied Health Literature (CINAHL); ProQuest (all databases); and Medline. A manual search of the identified articles was also undertaken to identify further relevant articles. The search found 240 peer reviewed articles related to QOL for PWD, with articles generated from the USA; Canada; Australia; UK, Netherlands; Belgium; Scandinavia; and Asia. The intent was to create a broad and inclusive database of relevant papers in order to support consideration of emerging areas of interest reflected in contemporary literature. This review does not, however, claim to be exhaustive.

\section{RESULTS}

Perhaps the most revealing aspect of this review of the literature was the volume and diversity of work being undertaken in relation to the QOL of PWD. This diversity was evident in topics ranging from architecture to wound care and is due, in part, to the growing use of $Q O L$ as an outcome measure in intervention research with PWD. Add to this the conceptual and clinical papers published over the same period and one has a picture of a vibrant research and clinical domain.

Despite this, it is still argued that little is known about the QOL of people living with dementia today (Selwood, Thorgrimsen \& Orrell, 2005; Hoe et al. 2006). In part, this is due to growing awareness and broadening of the way we conceptualise and consider QOL for PWD (O'Connor et al. 2009). It is also, in some part, due to the challenges associated with researching and measuring something that is uniquely individual (Carr \& Higginson, 2001). 


\section{Evolution}

Despite these challenges, much of the literature on QOL for PWD is devoted to its assessment and measurement, however, even this significant body of work has evolved markedly over the last decade. Indeed, the rapid shift and evolution of understandings, conceptualisation and measurement of all aspects of QOL for PWD is the overriding theme of this review.

In relation to the measurement of $\mathrm{QOL}$ for $\mathrm{PWD}$, this evolution has seen both a focusing and a broadening of approaches to assessing QOL for both clinical and research purposes. For example, early instruments measuring QOL largely focused on cognitively intact older populations, often because of logistical, conceptual and measurement difficulties (Brod et al. 1999; Moyle et al. 2007). Over time, disease specific instruments have been developed (Brod et al. 1999; Logsdon et al. 1999; Trigg, Jones et al. 2007; Trigg, Skevington et al. 2007) and tested for ever-more specific populations (Thompson \& Kingston, 2004; Ready, Ott \& Grace, 2004 \& 2006; Hoe et al. 2006; Trigg et al. 2007); locations (Kane et al. 2003; Hoe et al. 2006); and cultures (Missotten et al. 2008; Yap et al. 2008). A number of excellent literature reviews and evaluations of these various tools already exist (Walker, Salek, \& Bayer, 1998; Courtney et al. 2003; Thompson \& Kingston, 2004; Schölzel-Dorenbos et al. 2007; Banerjee et al. 2009) and an indepth analysis of these instruments is outside the remit of this review.

This increasing focus has resulted in a growing body of work devoted to the evaluation of patient versus proxy reports in relation to QOL for PWD (Boyer et al. 2004; Ready et al. 2004 \& 2006; Hoe et al. 2006; Vogel et al. 2006; Hoe et al. 2007; Kolanowski, Hoffman \& Hofer, 2007; Trigg, Jones et al. 2007; Arlt et al. 2008). These studies suggest that there are often differences between PWD and proxy evaluations in relation to $\mathrm{QOL}$, with PWD frequently rating their $\mathrm{QOL}$ higher than proxies. Possible reasons for this variation are identified in the literature, including the 'disability paradox' (Carr \& Higginson, 2001); anosognosia (Vogel et 
al. 2006); and adaptive capabilities common among those with chronic diseases (Ettema et al. 2005). It is also suggested that PWD and caregivers give weight to very different domains when assessing their / other's QOL (Murray \& Boyd, 2009).

As a result of this, there has also evolved a broadening of the focus and conceptions of QOL for PWD. Researchers are increasingly calling for a variety of sources of QOL data to be considered (Banerjee et al. (2009), including QOL measurement with both proxies and patients (Ready et al. 2004 \& 2006; Logsdon et al. 1999; Arlt et al. 2008); observation (Barnes, 2006); and interviews (Hubbard, Downs \& Tester, 2003; Steeman et al. 2007). Bond (1999) suggests a 'pluralistic approach' to QOL assessment, with involvement and input from PWD, family caregivers and researchers, as well as awareness of the different interpretations and assessments of QOL underpinned by various perspectives on dementia (eg, biomedical, psychological and social models).

\section{Dialogue: The Voice of People with Dementia}

Indeed, the last decade has seen a rise in research exploring the meaning of the condition for PWD (Bond, 1999) and on hearing the voice of the person with dementia (Cahill et al. 2004; Moyle et al. 2007; O'Connor et al. 2007; Ryan et al. 2008). This is largely aligned to a growing awareness of, and attempts to operationalise concepts of personhood and person- and relationship-centred care. It is also reflective of calls for a more 'meaningful dialogue' and in-depth understanding of the experiences and perceptions of people with dementia, particularly as it relates to their perceived QOL.

In general, this approach seeks to challenge notions of dwindling personhood and QOL in PWD (Moore \& Hollett, 2003). It is underpinned by individualised notions of QOL. While recognising some universal aspects of QOL, individual QOL considers individual weightings - that is, some aspects / domains may be more important than others to the individual. This variability may be influenced by 
generic and individual factors and their interaction over time (Carr \& Higginson, 2003). In this sense, QOL is dynamic and relational.

In line with this, much of the research in this area is qualitative in nature predominantly ethnographic (eg. Hubbard et al. 2003) and grounded theory (eg. Steeman et al, 2007), or mixed methods (Cahill et al. 2004; Moyle et al. 2007). Hearing the voice of the person with dementia is not without challenges however. Moore and Hollett (2003) identify a number of methodological challenges for researchers in this area, including the need to modify consent / assent procedures; sample size; facilitate communication; and make the most of the data. Importantly, they note the need to respect the dignity and personhood of people with dementia during this type of research. Hubbard et al. (2003) call for researchers to go beyond merely giving consideration to the effect of dementia in locating the experience of the person within the totality of their life. They call for flexibility; timing; preliminary meetings; and bespoke methods for each person with dementia. Increasingly, such bespoke methods are seen to reflect the uniqueness of the person and are associated with human values and rights, such as dignity and respect.

\section{Dignity}

Given the focus of other papers in this section, this review will not delve too deeply into the association of dignity and QOL in dementia, other than to note that many of the conceptual domains or constructs associated with QOL at the philosophical and theoretical level are increasingly receiving more attention in conceptual and research literature. Among these, autonomy, choice and control and, in particular, dignity, are increasingly linked to notions of personhood and QOL (Koppelman, 2002; Nay, 2002; Barnes, 2003; Boyle, 2008; Dwyer et al. 2009). As such, this body of literature reflects the growing awareness of personhood as an essential and foundational element in QOL. 
Dignity is associated with notions of self-worth and human agency and is defined as "both a feeling experienced by residents (eg. dignity being respected) and a status conferred on residents (eg. residents allowed their dignity) (Kane et al. 2003, p. 241). As such, it is inherently relational and interactional. Dignity may be found in risk (Nay, 2002); in specialised care (eg. palliative care [Volicer, 2007]; in staff issues (Dwyer et al. 2009); environment and space (Barnes, 2006); and in questions of capacity, self and decision-making for people with dementia (Koppelman, 2002). It is also linked to care and living practices, such as those associated with food, meal times and meal spaces (Barnes, 2006; Robinson \& Gallagher, 2008).

\section{Dining: Food and Meal Practices}

Interestingly, the search of the literature also revealed papers from the fields of nutrition and dietetics. These papers generally associated food, meal times and meal practices with QOL through their impact on dignity and autonomy, and the social and nurturative aspects of food (eg. Vogelzang, 2003). Vogelzang (2003) notes food is unique in that it meets a variety of needs beyond just the physical need to fuel the body. It is also associated with memory (both taste and smell) and may be imbued with social significance (eg. birthday cake, special restaurants or meals). In this sense, food is intrinsically linked to emotions. Food and meal times may also be considered a form of therapy, in both a reminiscent, as well as functional / meaningful activity way (Vogelzang, 2003). In this sense, food, particularly food preparation and meal time activities, is also engaging and active.

Indeed, mealtimes in group living situations, such as in nursing and care homes offers an opportunity to implement both physical and social care to enhance QOL (Nijs, de Graaf, Kok \& van Staveren, 2006). For many PWD, food may remain an enduring pleasure, and meal time may foster opportunities for socialisation, satisfaction, self-fulfilment and enjoyment (Robinson \& Gallagher, 2008). Importantly, it may also be one aspect of life over which they can maintain some 
control (Robinson \& Gallagher, 2008). Robinson and Gallagher (2008) suggest that the dining environment, timing of meals, menus and caregiver approaches and interactions are all amenable to manipulation and intervention, and offer opportunities to move away from traditional rules, routines and regulation-based care towards a dignified, person-centred approach.

While many of the articles written on food and meals were clinically focused discussions offering novel and thoughtful ways to integrate therapeutic activity, food, personhood and dignity, there were few research based articles. One such research article presented the findings of a randomised control trial on the effect of family-style mealtimes on QOL for older people in Dutch nursing homes and found improvements in residents' self reported QOL. Unfortunately, this study did not include PWD (Nijs et al. 2006) although the authors did draw links to earlier research that focused on PWD (Goddaer \& Abraham, 1994; Regneskog et al. 1996) and concluded that their findings may well extend to all nursing home residents, including those with cognitive impairment. This is in keeping with the results of Altus, Engelman and Mathew's (2002) study that concluded that family-style meals had a positive impact on participation and communication among PWD living in nursing and care homes. The association of communication and participation with social engagement and identity suggests that such findings may also influence perceived QOL (Altuset al. 2002; Murray \& Boyd, 2009).

In this respect, the literature on dining and food mirrors the evolution of dementia QOL literature generally in expanding the focus on objective measurement and intervention research to include broader and more individual, philosophical considerations associated with QOL, such as dignity and autonomy.

\section{DISCUSSION AND RECOMMENDATIONS}

QOL is a multifaceted, complex and highly individual concept. It may be conceptualised as an individual experience or a group outcome measure. Despite challenges in relation to the concept, QOL remains a focus of health 
research because, as with many other chronic degenerative conditions, health care providers have greater opportunity to intervene and affect QOL than to affect the course of the disease (Brod et al. 1999). QOL has become an important concept in clinical practice, health service research and policy making (Russell, Middleton \& Shanley, 2008), as well as in regulation, quality improvement and public information (Kane et al. 2003). QOL measures may be used to assess the quality of a service, the need for health care (Carr \& Higginson, 2001), the effectiveness of interventions (Carr \& Higginson, 2001; Schölzel-Dorenbos et al. 2007) and economic analysis (Selai \& Trimble, 1999).

As a result, much of the literature remains focused on the measurement and assessment of QOL for people with dementia from a domain, psychometric and evaluative stance. The literature is evolving however, to incorporate challenges and critiques in relation to broadening conceptions of $\mathrm{QOL}$ for people with dementia. An example of this is the debate around the use of proxy versus patient measures and the variability inherent in self versus third party assessments in conditions such as dementia regardless of degree of cognitive impairment (Thomson \& Kingston, 2004; Ready et al. 2006).

Despite this, there remains a strong focus on the development and testing of instruments to assess and measure QOL as an outcome measure for a variety of interventions. Improvements in QOL for PWD have a high priority in both care and research, and QOL assessment provides an important format for PWD and caregivers to "express whether an intervention has made an important difference to the patient's life" (Schölzel-Dorenbos et al. 2007, p. 511).

This is not to say that assessing and measuring QOL in an objective way is not challenging. A number of critiques have been posed in relation to measuring QOL in PWD (Carr \& Higginson, 2001; Silberfeld et al. 2002; Russell et al. 2008). These critiques question the use of standardised measures in what is essentially an individual experience, and argue that imposing preselected domains and 
standardised models measures general health status rather than individually conceived QOL (Carr \& Higginson, 2001). There are also issues associated with the link between QOL and quality of care (Russell et al. 2008); theoretical conceptualisation (Carr \& Higginson, 2001; Russell et al. 2008) and measurement challenges associated with the cognitive changes of dementia (Banerjee et al. 2009) and anosognosia (Vogel et al. 2006). Despite this, QOL does not necessarily decrease as cognition worsens (Hoe et al. 2006).

These challenges have led many researchers to broaden their focus and to link QOL understanding and measurement to principles of person- and relationshipcentred care. This has led to a growing body of work that considers QOL in a broad, yet individual way. Attention is turning to many of the individual rights or psychological domains, such as dignity, autonomy, choice and control, and the influence of these domains on care and life activities, such as those practices and activities associated with food and mealtimes.

This review of the literature related to QOL for PWD suggests that this is a vibrant area of discovery and development in terms of our thinking and our ability to measure and engage with what is essentially a very complex concept. This complexity underpins diversity in conceptualisations, approaches and methods.

A broad reading of the literature in this area highlights the potential for greater interdisciplinarity in both research and conceptual work. The breadth of QOL concepts supports the involvement of a range of disciplines, both health (medicine, nursing, allied health, health policy, nutrition, medical ethics) and nonhealth related (eg. economics, architecture and design, public policy). This interdisciplinarity also supports the development of new models of dementia care that weave person- and relationship-centred care principles and QOL together in ways that support best practice approaches and QOL for people with dementia. Finally, as Meyer and Owen (2008) and Robinson and Gallagher, (2008) note the key to achieving this is through culture change at all levels of care and social 
International Journal of Older People Nursing: QOL and Dementia: Practice Development Section

support for people with dementia. This starts with a greater understanding of dementia and a re-conceptualisation of QOL for this population. 


\section{REFERENCES}

Altus, D.E., Engelman, K.K. \& Mathews, R.M. (2002). Using family-style meals to increase participation and communication in persons with dementia. Journal of Gerontological Nursing, 28, 47 - 53.

Arlt, S., Hornung, J., Eichlaub, M., Jahn, H., Bullinger, M. \& Petersen, C. (2008). The patient with dementia, the caregiver and the doctor: cognition, depression and quality of life from three perspectives. International Journal of Geriatric Psychiatry, 23, $604-610$.

Banerjee, S., Samsi, K., Petrie, C.D., Alvir, J., Treglia, M., Schwam, E.M. \& del Valle, M. (2009). What do we know about quality of life? A review of the emerging evidence on predictive and explanatory value of disease specific measures of health related quality of life in people with dementia. International Journal of Geriatric Psychiatry, 24, 15 - 24.

Barnes, S. (2006). Space, choice and control, and quality of life in care settings for older people. Environment and Behaviour, 38(5), 589 - 604.

Birren, J., Lubben, J. \& Rowe, J. (1991). The Concept of Measurement of Quality of Life in the Frail Elderly. Academic Press: San Diego.

Bond, J. (1999). Quality of life for people with dementia: Approaches to the challenge of measurement. Ageing and Society, 19, 561 - 579.

Boyer, F., Novella, J., Morrone, I., Jolly, D. \& Blanchard, F. (2004). Agreement between dementia patient report and proxy reports using the Nottingham Health Profile. International Journal of Geriatric Psychiatry, 19, 1026 1034.

Boyle, G. (2008). Autonomy in long-term care: A need, a right or a luxury? Disability and Spirituality, 23(4), $299-310$.

Brod, M., Stewart, A.L., Sands, L. \& Walton, P. (1999). Conceptualization and measurement of quality of life in dementia: The dementia quality of life instrument (DQOL). The Gerontologist, 39(1), 25 - 35.

Cahill, S., Begley, E., Topo, P., Saarikalle, K., Macijauskiene, J., Budraitiene, A., Hagen, I., Holthe, T. \& Jones, K. (2004). "I know where this is going and I know it won't go back": Hearing the individual's voice in dementia quality of life assessments. Dementia, 3(3), 313 - 330.

Carr, A.J. \& Higginson, I.J. (2001). Are quality of life measures patient centred? British Medical Journal, 322, 1357 - 1360.

Courtney, M., Edwards, H., Stephan, J., O’Reilly, M. \& Duggan, C. (2003). Quality of life measures for residents of aged care facilities: A literature review. Australasian Journal of Nursing, 22(2), $58-64$.

Dwyer, L., Andershed, B., Nordenfeldt, L. \& Ternestedt, B. (2009). Dignity as experienced by nursing home staff. International Journal of Older People Nursing, 4, $185-193$.

Ettema, T.P., Dröes, R., de Lange, J., Ooms, M.E., Mellenbergh, G.J. \& Ribbe, M.W. (2005). The concept of quality of life in dementia in different stages of the disease. International Journal of Psychogeriatrics, 17(3), $353-370$.

Ferri, CP., Prince, M., Brayne, C., Brodarty, H., Fratiglioni, L., Ganguli, M., et al. (2005). Global prevalence of dementia: A delphi consensus study. Lancet, $366(9503), 2112-2117$. 
Goddaer, J. \& Abraham, I.L. (1994). Effect of relaxing music on agitation during mealtimes among nursing home residents with severe cognitive impairment. Archives of Psychiatric Nursing, 8, 150 - 158.

Hoe, J., Hancock, G., Livingston, G., \& Orrell, M. (2006). Quality of life of people with dementia in residential care homes. British Journal of Psychiatry, 188, $460-464$.

Hoe, J., Katona, C., Orrell, M. \& Livingston, G. (2007). Quality of life in dementia: Care recipient and caregiver perceptions of quality of life in dementia: the LASER-AD study. International Journal of Geriatric Psychiatry, 22, 1031 1036.

Hubbard, G., Downs, M.G. \& Tester, S. (2003). Including older people with dementia in research: challenges and strategies. Ageing and Mental Health, 7(5), $351-362$.

Kane, R.A., Kling, K.C., Bershadsky, B., Kane, R, L., Giles, K., Degenholtz, H.B., Liu, J. \& Cutler, L.J. (2003). Quality of life measures for nursing home residents. Journal of Gerontology, 58A(3), $240-248$.

Kolanowski, A., Hoffman, L., \& Hofer, S.M. (2007). Concordance of self-report and informant assessment of emotional well-being in nursing home residents with dementia. The Journals of Gerontology, 62B(1), $20-27$.

Koppelman, E.R. (2002). Dementia and dignity: Towards a new method of surrogate decision making. Journal of Medicine and Philosophy, 27(1), 65 -85 .

Lawton, M.P. (1983). The varieties of wellbeing. Experimental Aging Research, $9(2), 65-72$.

Logsdon, R.G., Gibbons, L.E., McCurry, S.M. \& Terri, L. (1999). Quality of life in Alzheimer's disease: Patient and caregiver reports. Journal of Mental Health and Aging, 5, 21 - 32.

Meyer, J. \& Owen, T. (2008). Calling for an international dialogue on quality of life in care homes. International Journal of Older People Nursing, 3, 291 294.

Missotten, P., Squelard, G., Ylieff, M., Di Notte, D., Paquay, L., De Lepeleire, J. \& Fontaine, O. (2008). Quality of life in older Belgian people: comparisons between people with dementia, mild cognitive impairment, and controls. International Journal of Geriatric Psychiatry, 23, 1103 - 1109.

Moyle, W., McAllister, M., Venturato, L. \& Adams, T. (2007). Quality of life and dementia: The voice of the person with dementia. Dementia, 6(2), $175-$ 191.

Moore, T.F. \& Hollett, J. (2003). Giving voice to persons living with dementia: The researcher's opportunities and challenges. Nursing Science Quarterly, 16(2), $163-167$.

Murray, L.M. \& Boyd, S. (2009). Protecting personhood and achieving quality of life for older adults with dementia in the U.S. health care system. Journal of Aging and Health, 21(2), $350-373$.

Nay, R. (2002). The dignity of risk. Australian Nursing Journal, 9(9), 33.

Nijs, K., de Graaf, C., Kok, F.J., \& van Staveren, W.A. (2006). Effects of family 
style mealtimes on quality of life, physical performance, and body weight of nursing home residents: cluster randomised control trial. British Medical Journal, 332(7551), $1180-1184$.

O’Connor, D., Phinney, A., Smith, A., Small, J., Purves, B., Perry, J., Drance, E., Donnelly, M., Chaudhury, H. \& Beattie, L. (2009). Personhood in dementia care: Developing a research agenda for broadening the vision. Dementia, $6(1), 121-142$.

Ragneskog, H., Brane, G., Karlsson, I. \& Kihlgren, M. (1996). Influence of dinner music on food intake and symptoms common in dementia. Scandinavian Journal of Caring Sciences, 10, $11-17$.

Ready, R.E., Ott, B.R. \& Grace, J. (2004). Patient versus informant perspectives of quality of life in mild cognitive impairment and Alzheimer's disease. International Journal of Geriatric Psychiatry, 19, 256 - 265.

Ready, R.E., Ott, B.R. \& Grace, J. (2006). Effects of quality of life reports from mild cognitive impairment and Alzheimer's disease patients. American Journal of Alzheimer's Disease and Other Dementias, 21(4), 242 - 248.

Robinson, G.E. \& Gallagher, A. (2008). Culture change impacts quality of life for nursing home residents. Topics in Clinical Nutrition, 23(2), 120 - 130.

Russell, C., Middleton, H. \& Shanley, C. (2008). Dying with dignity: The views of family caregivers about quality of life. Australasian Journal on Ageing, 27(2), $89-92$.

Ryan, T., Nolan, M., Reid, D. \& Enderby, P. (2008). Using the Senses Framework to achieve relationship-centred dementia care services. Dementia, 7(1), $71-95$.

Schölzel-Dorenbos, C.J.M., Ettema, T.P., Bos, J., Boelens-vannder Knoop, E., Gerritsen, D.L., Hoogeveen, F., de Lange, J., Meihuisen, L. \& Dröes, R. (2007). Evaluating the outcome of interventions on quality of life in dementia: Selection of the appropriate scale. International Journal of Geriatric Psychiatry, 22, $511-519$.

Selai, C. \& Trimble, M.R. (1999). Assessing quality of life. Ageing and Mental Health, 3(2), $101-111$.

Selwood, A., Thorgrimsen, L. \& Orrell, M. (2005). Quality of life in dementia: A one-year follow-up study. International Journal of Geriatric Psychiatry, 20, $232-237$.

Silberfeld, M., Rueda, S., Krahn, M. \& Naglie, G. (2002). Content validity for dementia of three generic preference based health related quality of life instruments. Quality of Life Research, 11, $71-79$.

Steeman, E., Godderis, J., Grypdonck, M., De Bal, N. \& Dierckx De Casterle, B. (2007). Living with dementia from the perspective of older people: Is it a positive story? Aging and Mental Health, 11(2), 119 - 130.

Thompson, L. \& Kingston, P. (2004). Measures to assess the quality of life for people with advanced dementia: Issues in measurement and conceptualisation. Quality in Ageing, 5(4), 29 - 39.

Trigg, R., Jones, R.W. \& Skevington, S.M. (2007). Can people with mild to moderate dementia provide reliable answers about their quality of life? Age and Ageing, 36, $663-669$.

Trigg, R., Skevington, S.M. \& Jones, R.W. (2007). How can we best assess the 
quality of life if people with dementia? The Bath Assessment of Subjective Quality of Life in Dementia (BASQID). The Gerontologist, 47(6), 789 797.

Vogel, A., Mortensen, E.L., Hasselbalch, S.G., Andersen, B.B. \& Waldemar, G. (2006). Patient versus informant reported quality of life in the earliest phases of Alzheimer's disease. International Journal of Geriatric Psychiatry, 21, 1132 - 1138.

Vogelzang, J.L. (2003). Dignity and dietary interventions for dementia. Home Healthcare Nurse, 21(1), $40-42$.

Volicer, L. (2007). Goals of care in advanced dementia: Quality of life, dignity and comfort. The Journal of Nutrition, Health and Aging. 11(6), 481.

Walker, A. \& Lowenstein, A. (2009). European perspectives on quality of life in old age. European Journal on Ageing, 6, 61-66.

Walker, M., Salek, S. \& Bayer, A. (1998). A review of quality of life in Alzheimer's disease: Issues in assessing the disease impact. Pharmacoeconomics, 14(5), $499-430$.

World Health Organisation.(2003). World Health Report 2003: Shaping the Future. World Health Organisation: Geneva.

WHOQOL Group. (2004). Introducing the WHOQOL Instruments. World Health Organisation: Geneva.

Yap, P., Goh, J., Henderson, L.M., Han, P.M., Ong, K.S., Kwek, S., Ong, E., Loh, D. (2008). How do Chinese patients with dementia rate their own quality of life? International Psychogeriatrics, 20(3), 482 - 493. 


\section{Revisions}

\begin{tabular}{|c|c|}
\hline Recommendation & Action \\
\hline $\begin{array}{l}\text { Reviewer } 1 \text { : The abstract seems very } \\
\text { brief. Having reviewed the paper, you } \\
\text { could put the } 3 \text { Ds into context to prepare } \\
\text { readers (especially why dining appears) } \\
\text { and refer to the emphasis on } \\
\text { assessment and measurement of QoL } \\
\text { here. You could add in more key words } \\
\text { The } 3 \text { Ds are not included. } \\
\text { Reviewer } 3 \text { : Revisit the abstract } \\
\text { conclusion. It is not clear what is mean } \\
\text { by the final sentence }\end{array}$ & $\begin{array}{l}\text { The Abstract has been revised in line with } \\
\text { reviewers' } 1 \text { and } 3 \text { comments. }\end{array}$ \\
\hline $\begin{array}{l}\text { Reviewer } 1 \text { : It would be useful if you } \\
\text { identify how many papers you did review, } \\
\text { their geographic spread and any other } \\
\text { relevant factors. A table could help } \\
\text { readers. }\end{array}$ & $\begin{array}{l}\text { The number of papers reviewed has been } \\
\text { included, as has the geographic spread. } \\
\text { A table has not been included due to the } \\
\text { number of papers reviewed. }\end{array}$ \\
\hline $\begin{array}{l}\text { Reviewer 2: Remove reference to } \\
\text { Australia - it begs the question 'what } \\
\text { about UK, US, Asia.... }\end{array}$ & $\begin{array}{l}\text { Reference to Australia has been removed } \\
\text { from the Introduction. }\end{array}$ \\
\hline $\begin{array}{l}\text { Reviewer 3: Indicate QOL as referring to } \\
\text { quality of life in page } 2\end{array}$ & $\begin{array}{l}\text { The abbreviation (QOL) has been added } \\
\text { on page } 2 \text {. }\end{array}$ \\
\hline $\begin{array}{l}\text { Reviewer 3: Revisit the use of THAT } \\
\text { throughout paper as there are examples } \\
\text { where THAT is not needed }\end{array}$ & $\begin{array}{l}\text { Grammatical errors addressed by both } \\
\text { author and professional editor. }\end{array}$ \\
\hline $\begin{array}{l}\text { Reviewer 3: Revisit referencing } \\
\text { throughout the paper and in reference list } \\
\text { to ensure it meets journal requirements }\end{array}$ & $\begin{array}{l}\text { References reviewed and amended in } \\
\text { line with journal requirements. }\end{array}$ \\
\hline $\begin{array}{l}\text { Reviewer 3: Revisit aims (p.4) to indicate } \\
\text { the type of literature reviewed. }\end{array}$ & $\begin{array}{l}\text { Aims and Method section have been } \\
\text { revised and expanded to justify the } \\
\text { inclusion of conceptual and clinical, as } \\
\text { well as research literature in this review. }\end{array}$ \\
\hline $\begin{array}{l}\text { Reviewer 3: Why did the literature review } \\
\text { involve contemporary literature } \\
\text { (research, AND discussion AND } \\
\text { philosophy papers?). Page } 2 \text { indicates } \\
\text { the review is focused on work directed } \\
\text { towards QOL for people living with } \\
\text { dementia - it is not clear what the work } \\
\text { is, ie research or otherwise and the } \\
\text { benefit of including discussion papers in } \\
\text { this review }\end{array}$ & $\begin{array}{l}\text { The review is indentified as a broad } \\
\text { consideration of the area of QOL for PWD } \\
\text { (see above re: Aims and Method) } \\
\text { Language changed - papers are } \\
\text { identified as conceptual, clinical and } \\
\text { research based (rather than } \\
\text { philosophical, discussion and research } \\
\text { based) } \\
\text { Page 2: amendment and clarification } \\
\text { made regarding the type of work included } \\
\text { in this review. }\end{array}$ \\
\hline $\begin{array}{l}\text { Reviewer 3: } p 11 \text {, line } 39 \text { should be AS } \\
\text { rather than 'a ell as in regulation... } \\
\text { Add as to } p 13 \text { lin } 4 \text {, ie '... care and life } \\
\text { activities, such AS those }\end{array}$ & Identified typographical errors corrected. \\
\hline
\end{tabular}

